\title{
Nitrogen Mustard Compound
}

National Cancer Institute

\section{Source}

National Cancer Institute. Nitrogen Mustard Compound. NCI Thesaurus. Code C697.

A mustard agent containing nitrogen and chlorine atoms. 